\title{
Article \\ An Enhanced Heterogeneous Gateway-Based Energy-Aware Multi-hop Routing Protocol for Wireless Sensor Networks
}

\author{
Fuseini Jibreel ${ }^{1}$, Emmanuel Tuyishimire ${ }^{2}$, M. I. Daabo ${ }^{3}$ \\ ${ }^{1}$ Tamale Technical University \\ ${ }^{2}$ University of Cape Town; emmanuel.tuyishimire@uct.ac.za \\ ${ }^{3}$ Department of computer science, University For Development Studies Ghana \\ * Correspondence: emmanuel.tuyishimire@uct.ac.za
}

\begin{abstract}
Wireless Sensor Networks (WSNs) continue to provide essential services for various applications such as surveillance, data gathering, and data transmission from the hazardous environments to safer destinations. This has been enhanced by the energy-efficient routing protocols that are mostly designed for such purposes. Gateway-based Energy-Aware Multi-hop Routing protocol (MGEAR) is one of the homogenous routing schemes that was recently designed to more efficiently reduce the energy consumption of distant nodes. However, it has been found that the protocol has a high energy consumption rate, lower stability period, less data transmission to the Base station (BS). In this paper, an enhanced Heterogeneous Gateway-based Energy-Aware multi-hop routing protocol ( HMGEAR) is proposed. The proposed routing scheme is based on the introduction of heterogeneous nodes in the existing scheme, selection of the head based on the residual energy, introduction of multi-hop communication strategy in all the regions of the network, and implementation of energy hole elimination technique. Results show that the proposed routing scheme outperforms two existing ones.
\end{abstract}

Keywords: wireless sensor networks; heterogeneous; hazardous environment; energy efficient.

Citation: Lastname, F.; Lastname, F.; Lastname, F. Title. Preprints 2021, 1, 0. https://doi.org/

Received:

Accepted:

Published:

Publisher's Note: MDPI stays neutral with regard to jurisdictional claims in published maps and institutional affiliations.

\section{Introduction}

We are in era when the Internet of things (IoT) become a popular response to various technological challenges [1-3]. RFID tags are commonly attached (or embedded) to things and wirelessly communicate to achieve certain goals. This creates a complex wireless networks on which communication models are still a subject of research. Furthermore, WSNs have been deployed in various environments to assist moving objects such as drones and robots to perform various tasks [4,5].

The IoT, as a WSN, since their inception, provide critical assistance to mankind especially in gathering information from the hazardous environment. Indeed, the networks have the ability to collect, process, and transmit data from critical environmental conditions to safer places. These are some of the reasons why they are now being used in various applications such as in agriculture, healthcare, environmental monitoring, military surveillance, structural monitoring, traffic control, and river level variation monitoring, etc.[6].

When data is collected in these networks, it is sent to a destination node called the Base Station (BS) for further processes. These networks are usually made up of tiny sensor nodes. For the source of energy, the sensor nodes rely on rechargeable and replaceable batteries. However, charging and replacing these batteries are practically impossible when they are deployed in a hazardous environment. Therefore, the efficient use of the limited resources of the sensor nodes always helps to improve the performance of the network. The performance of a WSN can be improved by developing mechanisms that can reduce the energy depletion of the nodes and extend the lifetime of the network. The various units of the sensor nodes where the amount of energy is being used are the sensing, data processing, and communication units. The communication unit is where the highest energy consumption is observed [7]. To minimize energy consumptions in these units many energy preservation techniques such as clustering, data aggregation, effective node deployment, and cluster-based routing protocols, etc. have been developed. 
Hierarchical routing protocols in WSNs are seen as the most energy-efficient schemes that have been widely used in WSNs [8]. Any of the schemes divides the network into groups called clusters with each cluster having a head called a central node called Cluster Head $(\mathrm{CH})$. These heads receive the sensed data from the local nodes, aggregate the data and report to the Base Station (BS) through a single-hop or multi-hop communication approach depending on the distance from the BS. An example of such routing models can be found in $[9,10]$.

In the related literature, there are several cluster-based routing algorithms that have been proposed in wireless sensor networks. One of the most important cluster-based routing protocols for wireless sensor network has been proposed in [11]. The protocol has become the basis for several cluster-based routing protocols in literature. The scheme operates in rounds and each round is sub divided into two phases namely: setup phase and steady state phase. In the setup phase each node produces a random number between 0 and 1 and once this random number is less than a particular threshold value $\mathrm{T}$ (n) which is given by Equation 1, then the node qualifies as a cluster head for the current round. After the $\mathrm{CH}$ election, non $\mathrm{CH}$ nodes select a $\mathrm{CH}$ based on the head's signal strength to be part of its cluster. These non $\mathrm{CH}$ then transmit the captured data through single-hop communication technique to the $\mathrm{CH}$ for aggregation and onward submission to the BS.

$$
T(n)= \begin{cases}\frac{P}{1-P\left(\operatorname{rmod}\left(\frac{1}{p}\right)\right)} & \text { if } n \in G \\ 0 & \text { otherwise }\end{cases}
$$

Where $r$ is the current round, $G$ is the set of nodes that are not selected as cluster head and $P$ is the desired percentage of cluster head.

The authors in [12] explained a probability-based clustering algorithm called Distributed Energy-Efficient Clustering algorithm (DEEC). The algorithm elects cluster heads based on the ratio between the residual energy of each node and the average energy of the network. However, the single-hop communication approach is used in sending data to the BS. This makes the distant nodes dissipate a huge amount of energy thereby affecting the lifetime of the network. In [13], Sharma and Verma have analyzed the Low Energy Adaptive Clustering Hierarchy (LEACH) protocol which is a homogeneous system and then studied the impact of heterogeneity. The authors then proposed a LEACH heterogeneous system which seeks to compare two systems; the heterogeneous and homogeneous systems. Simulation results using MATLAB shows that the proposed LEACH heterogeneous system significantly reduces energy consumption and increases the total lifetime of the wireless sensor network. The main problem identified in this scheme is that the cluster heads $(\mathrm{CHs})$ are not chosen based on their residual energy and this affects the lifetime of the network. A model called Developed Distributed Energy-Efficient Clustering (DDEEC) for heterogeneous wireless sensor networks has been proposed in [14]. It is the enhanced version of DEEC. This scheme resolves the penalizing effect in the DEEC protocol. However, the DDEEC has a similar problem as DEEC where a single-hop communication approach is used in sending data to the BS in the network. This makes the distant nodes dissipate a huge amount of energy thereby affecting the lifetime of the network.

An Enhanced Distributed Energy Efficient Clustering Scheme (EDEEC) for heterogeneous WSN has been described in [15]. This is an extended version of DEEC with normal, advanced, and super-node classification based on the node's energy. The problem identified in this protocol is similar to that of DEEC, SEP, and DDEEC in which no appropriate communication approach was introduced to reduce the energy depletion of the distant nodes deployed in the network.

In [16], the authors proposed a new optimization scheme. The new algorithm modified the average probability of advanced nodes whose residual energy is less than the Threv (threshold residual energy value) to now depend on the average distance of the nodes from the Base station rather than the average energy of the network. The scheme further implemented TEEN and different amplification energy levels in the protocol to conserve 
energy in the network. Results showed that the proposed protocol performed better than the existing scheme in terms of throughputs, residual energy and network lifetime. However, the single-hop communication technique used affected the lifetime of the distant nodes.

An improved form of E-DEEC has been proposed in [17]. iE-DEEC improved the election probability of the protocol in [8] by taking into account the distance of super-nodes and the average distance of all the nodes to the BS in selecting CHs. The scheme has also introduced different amplification energy levels to minimize the energy consumption during the communications between the $\mathrm{CHs}$ and BS and also within inter and intra clusters. MATLAB R2017a was used for simulation to evaluate the effectiveness of the scheme. The simulation results showed that the proposed protocol performed better than E-DEEC in terms of throughputs, residual energy and network lifetime.

Jibreel, in [18], explained an extended form of Threshold Stable Election Protocol called eTSEP. The new scheme introduces the distance and residual energy into the election probabilities of each level of the nodes. This allows nodes with high residual energy and closer to the Base station to stand a better chance of becoming a cluster head. The performance of the scheme was evaluated using MATLAB R2017a and compared with TSEP. It has been shown that the new protocol performed better than TSEP in terms of throughputs, residual energy and the network lifetime. However, the single-hop communication technique is used in data transmission from the CHs to the BS. This depletes the energy of the distant nodes.

It has been presented in [19] a heterogeneous form of Modified Low Energy Adaptive Clustering Hierarchy, Servant-MODLEACH (S-MODLEACH). The algorism uses three levels of nodes namely, advanced, servant and normal nodes. The protocol chooses cluster heads based on their residual energy and assigned data aggregation role to a group of nodes called servant nodes. It has been showed that S-MODLEACH achieved better outcomes than MODLEACH in respect of throughputs and the network lifetime. Although, the scheme used a multi-hop communication approach to reduce the energy depletion of the distant nodes, no mechanism was put in place to reduce energy holes created in the network.

An enhanced form of Threshold Distributed Energy Efficient Clustering protocol (TDEEC) has been proposed in [20]. The algorithm, Gateway based-TDEEC, introduced a gateway node at the middle of the sensing area and then installed the BS far away from the sensing field. The cluster heads relay their data to the gateway which will then aggregate the data and then send the final report to the BS. Results showed that, the proposed protocol outperformed the TDEEC in terms of stability period, throughput, residual energy and the network lifetime. It, however, did not consider a multi-hop communication strategy to reduce the energy expenditure of the distant nodes as well as reducing the energy holes created in the system.

In [21], it has been proposed a modified heterogeneous routing protocol called Distance-DEEC (D-DEEC). The protocol took into consideration the residual energy, distance of the individual nodes and average distance of all the nodes from the base station in selecting the cluster heads. This has allowed the scheme to select cluster heads with high residual energy, closer to the Base station and their neighbours. The performance of the proposed algorithm was evaluated using MATLAB R2017a and the outcomes showed that the D-DEEC protocol outperformed DEEC in terms of energy consumption, throughputs and the network lifetime.

The author, in [15], proposed TEEN-MGEAR(T-MGEAR). The scheme modified the election probability by considering the distance and the residual energy of the nodes in selecting the cluster heads. It has also employed hard and soft thresholds to determine when nodes can transmit their sensed data. no heterogeneity and multi-hop communication technique has been employed in this scheme.

In [22], a proposed Enhanced M-Gear Protocol for Lifetime Enhancement in Wireless Clustering System. In this scheme, the network was divided into a number of sections 
with each section having its own gateway node. The nodes capture data and transmit it to the gateway in their immediate section for onward submission to the next gateway or to the BS. The results showed that, it outperforms MGEAR in terms of throughput, energy consumption and network lifetime. However, having several gateways will lead to an increase in the cost of the network and also, the absence of heterogeneous nodes affects the stability period of the network.

The authors in [23] explained a Gateway- Stable Election Protocol (G-SEP). The G-SEP scheme altered the election probability of selecting the cluster heads by considering the distance, average distance and residual energy of the advanced nodes. The algorithm further introduced a gateway node at the middle of the network and then installed the BS outside the field. Simulation results using MATLAB R2017a showed that the G-SEP performs better than the Zonal-Stable Election protocol (ZSEP) in terms of coverage, stability period, and extension of the lifetime of the network.

Hence, having reviewed the literature related to increasing the network lifetime of WSNs, it is important to note that selecting CHs based on their residual energy of nodes, introducing heterogeneous nodes to strengthen the ability of nodes, introducing multi-hop communication technique to reduce the energy depletion of distant nodes and implementing energy holes removal mechanism to further conserve the energy of the nodes can remarkably improve the network lifetime.

This paper proposes a routing scheme by first proposing the an optimal network topology and secondly an enhanced routing algorithm is proposed. In fact in this paper, the $\mathrm{CH}$ s are selected based on their residual energy, heterogeneous nodes and multi-hop communication approach have been introduced since the effectiveness of this approach has proven in [23] and energy hole removal scheme has been implemented to enhance the lifetime of the network.

It is important to mention that this paper has been motivated by the work in [15]. Here, MGEAR has been improved using the original homogeneous nodes deployed in all the regions. In this model, the cluster heads are selected based on distance/ residual energy, because they were purely homogeneous nodes. This model has been complemented by the scheme proposed in [23], where the authors only employed a gateway node to the Stable Election Protocol (SEP) so that the BS can be repositioned outside the network. Here distance/average distance and residual energy were also considered while modifying SEP protocol. Here, it is only the homogeneous nodes that capture data, transmit it to the heads which are heterogeneous nodes of the same energy level to the gateway node to the base. No direct transmission has been employed in any part of this network. It has nothing to do with the model used in GMEAR. The proposed model complement the work done in the literature in the following four ways.

1. Heterogeneity has been introduced in the existing MGEAR: we have introduced heterogeneous nodes of different energy levels to the existing scheme especially in regions 3 and 4 which, to the best of our knowledge, was have been employed before.

2. Cluster heads are selected based on only residual energy because heterogeneous nodes are deployed in Regions 3 and 4 . These nodes give a better supporting role for long period and provide a better stability period than the homogeneous nodes deployed in the case of the models in $[15,23]$.

3. In the proposed scheme, both the direct and multi-hop communication technique have be employed from Region 4 to the BS which has not happened in $[15,23]$.

4. Energy hole elimination technique has been implemented to prevent nodes from losing their entire energy during data transmission which non of the models in $[15,23]$ has not considered.

The remainder of the paper is organized as follows. Section 2 described the proposed scheme, simulation results and analysis are discussed in Section 3 and the conclusion is then drawn in Section 4. 


\section{Proposed routing scheme}

In this section, the proposed heterogeneous protocol called HMGEAR is explained. This scheme builds on the homogeneous routing protocol suggested in [24].

In this scheme the network divides the sensor nodes into four logical regions on the basis of predefined threshold distance. The Base station and a gateway node are placed outside the sensing field and at the centre of the network respectively. The nodes whose distance from either the Base station or gateway node is less than the predefined distance, they are assigned in Regions 1 and 2. In that case, they transmit their data to either the Base station or gateway node using direct communication approach. These are the homogeneous nodes. However, if their distance is more than the predefined threshold distance and closer to the gateway node then they are placed in Region 3 else in Region 4 as illustrated in Figure 1. These are the heterogeneous nodes. Elections are conducted in these two regions and heads are selected based on the residual energy of the nodes. Data is transmitted to the BS through a multi-hop communication technique (see [25] for example) from these regions. The gateway node receives the final report from $\mathrm{CHs}$ in Region 3, aggregates the data before transmitting it to the BS. The nodes in each region transmit their data based on the energy threshold set out for them below which the nodes cannot transmit data but sleep to conserve their energies.

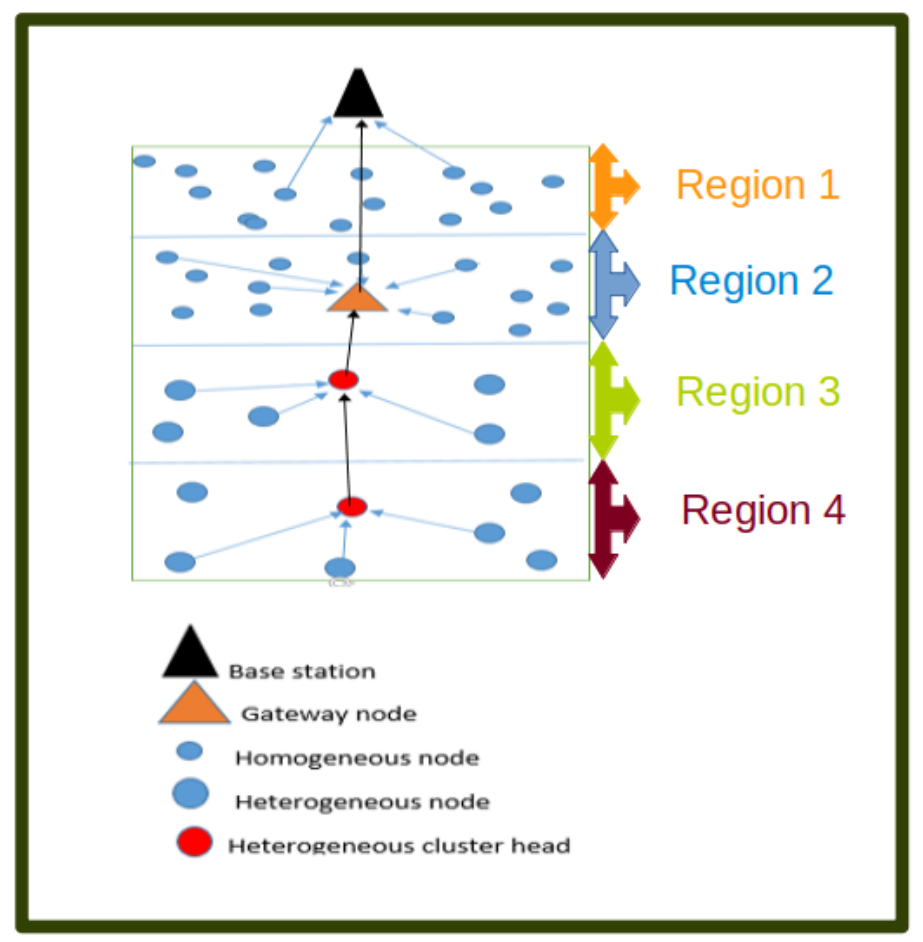

Figure 1. Proposed Network Model.

\subsection{Proposed network model}

The network model as depicted in Figure 1, is defined as follows. It is a network $G(B S, G W, H o, H e, L)$, where $B S$ is the network base station, $G W$ is the network gateway, $H o$ is the set of homogeneous nodes, $H e$ is the set of heterogeneous nodes and $L$ is the set of links connecting any of the defined nodes (any node in the union $B S \cup G W \cup H o \cup H e$ ).

The following are the network properties.

- The network can be subdivided in four subnetworks. located in Regions 1, 2, 3 and 4 as shown in Figure 1.

- There exists at least one node in Region 4 connected with a node in Region 3.

- The gateway is in Region 2 and is connected to the BS.

- The gateway is connected with the base station. 
- Each node in Region 1 is connected with the base. station.

- All nodes in Regions 3 and 4 are not connected with the base station.

\subsection{The routing scheme}

The proposed scheme is made up of two phases.

1. The setup phase. It consists of allocating nodes in 4 Regions as well as election of the cluster heads.

2. The steady phase. It consists on how data is transmitted among the nodes within the four regions. The energy involved in this data transmission is mostly determined in this phase.

\subsubsection{Setup Phase}

This session is similar to the setup phase suggested by the authors in [24]. The authors here, divided the homogeneous sensor nodes into four logical regions on the basis of their location in the sensing field. The base station is installed outside the sensing area and the gateway node is placed at the centre of the network. The nodes whose distance from either the Base station or the gateway node is less than the predefined distance threshold, transmit their data using direct communication techniques. The nodes whose distance is beyond the threshold distance are divided into equal regions. Elections of heads are conducted in these regions using the probability approach. These heads aggregate the data, transmit their report directly to the gateway node and then to the Base station.

The proposed scheme modified the election probabilities to take into account the residual energy of the nodes. The selection probabilities for the respective heterogeneous nodes in Region 3 and 4 are given by Equations 2 and 3.

$$
\begin{aligned}
& P_{\text {het } 1}=\left(\frac{P_{\text {opt }}(1+b)}{1+a m}\right) \frac{E_{i}}{E_{0}} \\
& P_{\text {het } 2}=\left(\frac{P_{\text {opt }}(1+a)}{1+a m}\right) \frac{E_{i}}{E_{0}}
\end{aligned}
$$

Where, $E_{i}$ is the residual energy of the node, $E_{0}$ is the initial energy of the node, $m$ is the percentage of sensor nodes equipped with $\mathrm{a}$ and $\mathrm{b}$ times more energy resources than the homogeneous sensor nodes in the network and $P_{\text {opt }}$ is the probability by which each node can become a $\mathrm{CH}$. However, $b=\frac{a}{2}$.

Their respective thresholds are also given in Equations 4 and 5 .

$$
T_{\text {het } 1}= \begin{cases}\frac{P_{\text {het } 1}}{1-P_{\text {het } 1}\left(\operatorname{rmod}\left(\frac{1}{p_{h e t 1}}\right)\right)} & \text { if } n \in H e \\ 0 & \text { otherwise }\end{cases}
$$

where $\mathrm{He}$ is the set of heterogeneous nodes that has not become a $\mathrm{CH}$ in the past $\frac{1}{P_{h e t 1}}$ round $r$ in Region 3.

$$
T_{\text {het } 2}= \begin{cases}\frac{P_{\text {het2 }}}{1-P_{\text {het2 }}\left(\operatorname{rmod}\left(\frac{1}{p_{\text {het } 2}}\right)\right)} & \text { if } n \in \text { Ho } \\ 0 & \text { otherwise }\end{cases}
$$

where $\mathrm{Ho}$ is the set of heterogeneous nodes that has not become cluster head in the past $\frac{1}{P_{\text {het } 2}}$ round $r$ in Region 4 . 


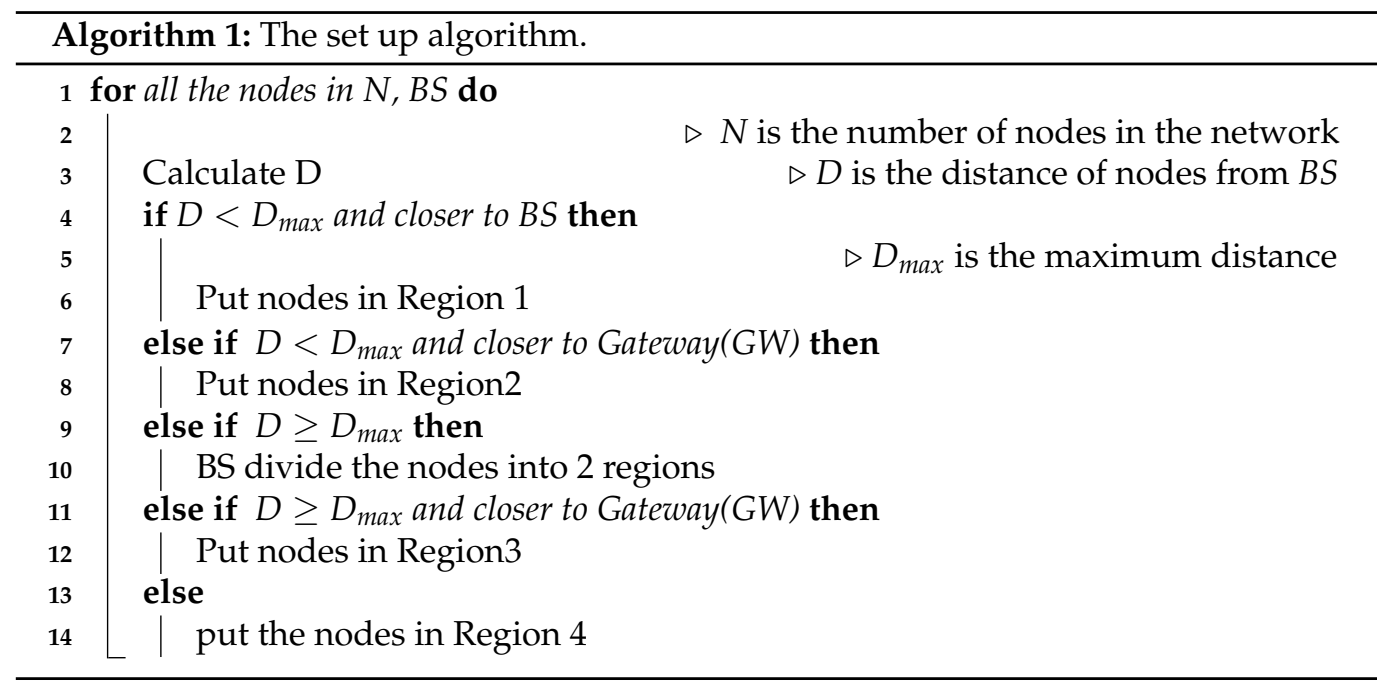

As shown by Algorithm 1, the proposed protocol assumes a network as specified in Subsection 2.1. As shown by the algorithm, Line 4-6, the homogeneous nodes which are closer to the BS and therefore communicate directly to BS are put in Region 1. Region 2 is considered to be where the gateway node is located, and it contains the homogeneous nodes. Once these nodes are also closer to the gateway node, they are considered to be in the region (see Lines 7-8). Regions 3 and 4 contain heterogeneous nodes. These nodes have higher energy than the homogeneous nodes placed in Regions 1 and 2 and therefore can sustain the energy of the network for a longer period of time despite their distance from the gateway nodes. Nodes are allocated to these two regions in Lines 9-14.

\subsubsection{Steady Phase}

In this section, the energies dissipated by the active sensor nodes in each region is determined and the total energy of the network is calculated. Applying the energy dissipation equation proposed by [26], the equations below are obtained. The energy $E_{n o n-B S}$ each low energy sensor in Region $1 R 1$ spent in relaying $k$ bits report to $B S$ is given by Equation 6 .

$$
E_{\text {non }-B S}=E_{T X}\left(K, d_{B S}\right)
$$

Where $d_{B S}$ is the distance between the homogeneous nodes in Region 1 and the BS.

Each low energy sensor in Region $2 R 2$ spent energy $E_{g w-B S}$ in relaying k bits report to the gateway node and is given by Equation 7 .

$$
E_{n o n-g w}=E_{T X}\left(K, d_{g w}\right)
$$

Where $d_{g w}$ is the distance between the homogeneous nodes in Region 2 and the gateway node.

The energy $E_{g w-B S}$ spent by the gateway node in receiving $k$ bits of data from Region 2 and 3, aggregating it, and relaying the report to the $B S$ is given by Equation 8 .

$$
E_{g w-B S}=k E_{\text {elect }}+k E_{\text {elect }}\left(\frac{n}{c}\right)+k\left(\frac{n}{c}\right) E_{D A}+E_{T X}\left(k, d_{B S}\right)
$$

Where $E_{\text {elect }}$ is the electronic energy of the transmitter, $n$ is the number of nodes deployed in the network $c$, the number of clusters in the network, and $E_{D A}$ energy for data aggregation.

The energy spent in receiving and relaying $\mathrm{k}$ bits report by the cluster head $\mathrm{CH} 1 \mathrm{in}$ Region 3 to the gateway node is given by Equation 9 . 


$$
E_{C H 1-g w}=k E_{\text {elect }}+k E_{\text {elect }}\left(\frac{n}{c}-1\right)+E_{T X}\left(k, d_{g w}\right)
$$

The energy spent in receiving and relaying $\mathrm{k}$ bits report from the cluster head $\mathrm{CH} 2 \mathrm{in}$ Region 4 to the cluster head $\mathrm{CH} 1$ in Region 3 is given by Equation 10.

$$
E_{\mathrm{CH} 2-\mathrm{CH} 1}=k E_{\text {elect }}\left(\frac{n}{c}-1\right)+E_{T X}\left(k, d_{C H 1}\right)
$$

Where $d C H 1$ is the distance between the cluster heads in Region 3 and 4 .

Total energy $E_{T}$ used in the network is given by Equation 11

$$
E_{T}=E_{n o n-B S}+E_{g w-B S}+E_{C H 1-g w}+E_{C H 2-C H 1}
$$

Elections of the cluster heads is conducted in Regions 3 and 4. As shown in Algorithm 2 , the election probabilities in the HMGEAR protocol are based on the residual energy of the nodes. So the node with high residual energy has a higher chance of becoming a cluster head. This is to delay the weak nodes which cannot transmit data effectively to the BS from becoming heads immediately. A multi-hop communication approach is introduced in these regions ( 3 and 4 ) between the two elected heterogeneous cluster heads before the final report reaches the gateway node which will then aggregate it before onward submission to the BS. This is to reduce the energy consumption of nodes in Region 4 which may be far from the region. Finally, Energy-efficient HOle Removing Mechanism (E-HORM) technique proposed in [24] is implemented. This technique finds the maximum distance nodes to calculate the maximum energy before data transmission. This maximum energy is referred to as a threshold energy $E_{t h}$. So each node will first check its energy level and if the energy level of the node is less than $E_{t h}$, it cannot transmit data. This helps to conserve energy. 


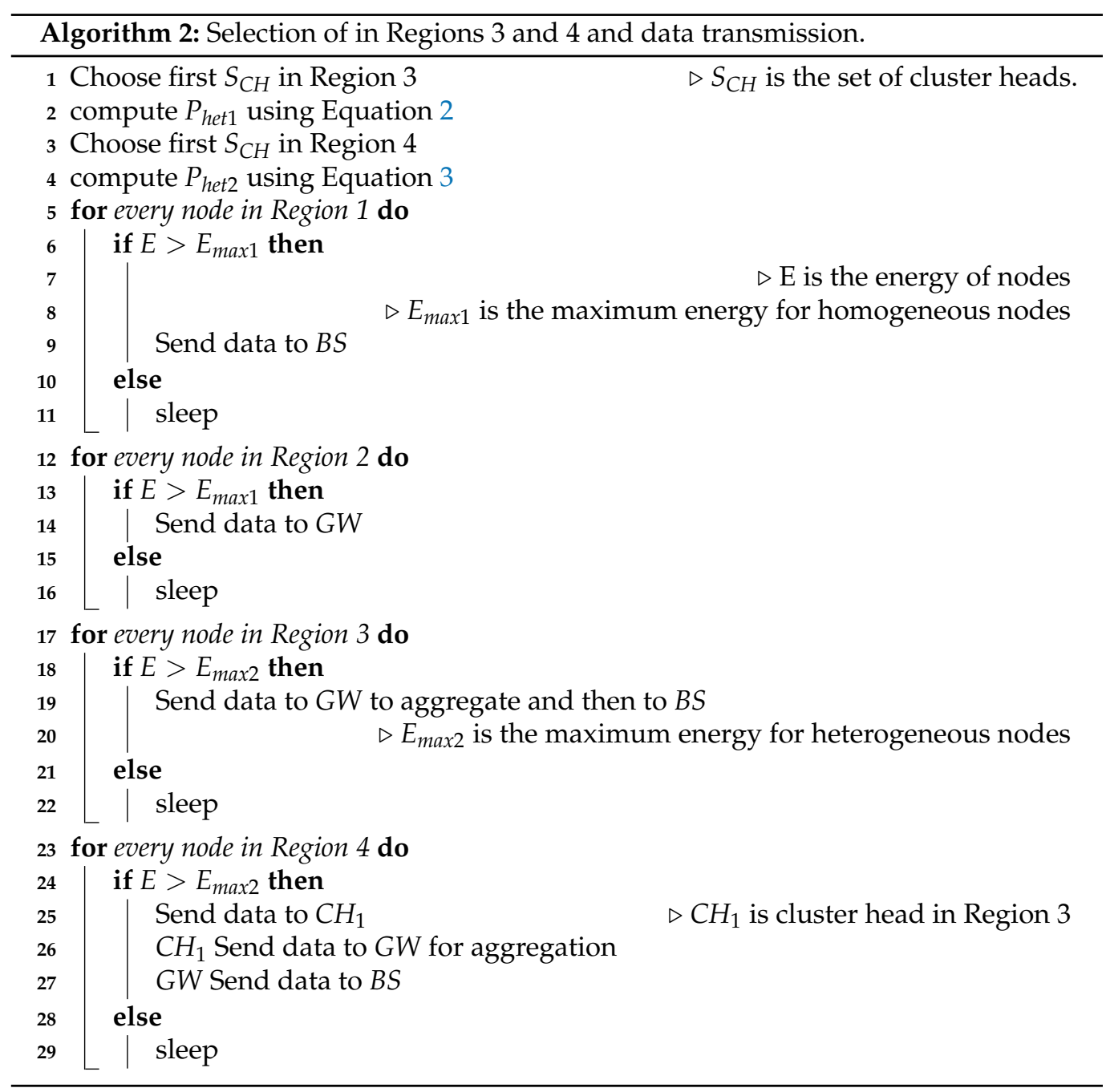

\section{Simulation results and analysis}

The proposed heterogeneous routing protocol was compared with the SEP and MGEAR routing protocols using MATLAB R2018a simulation. For the simulation, a system consisting of 100 sensor nodes was randomly deployed in a field of dimension $100 \mathrm{~m} \times 100 \mathrm{~m}$. The BS, as well as the gateway node, were respectively positioned at $(50 \mathrm{~m}$, $120 \mathrm{~m})$ and $(50 \mathrm{~m}, 50 \mathrm{~m})$ in the network. There are $20 \%$ of heterogeneous nodes which are prepared with greater energy than the homogeneous nodes $(m=0.2$ and $\alpha=1)$. All the nodes are stationary after deployment. 1 defines the simulation parameters used in this research work.

Table 1: Simulation Parameters.

\begin{tabular}{ll}
\hline Parameters & Values \\
\hline Network field & $100 \mathrm{~m} \times 100 \mathrm{~m}$ \\
\hline Number of nodes & 100 \\
\hline Initial energy of normal nodes $\left(E_{0}\right)$ & $0.5 \mathrm{~J}$ \\
\hline Message size & $4000 \mathrm{bits}$ \\
\hline$E_{\text {elec }}$ & $50 \mathrm{Nj} / \mathrm{bit}$ \\
\hline$E_{f s}$ & $10 \mathrm{Nj} / \mathrm{bit} / \mathrm{m}^{2}$ \\
\hline$E_{m p}$ & $0.0013 \mathrm{Pj} / \mathrm{bit}_{\mathrm{m}} \mathrm{m}^{2}$ \\
\hline$P_{\text {opt }}$ & 0.1 \\
\hline
\end{tabular}


The performance evaluation was done based on the following performance metrics as used in [11] and [26].

1. Number of alive nodes per cluster round: indicates the Number of nodes alive from the network for every cluster round and this depends on the availability of energy remaining in the network.

2. Number of dead nodes per cluster round: Number of nodes dead per cluster round as a result of changing energy level inside the network during network survival time. This also indicates the possible lifetime remaining of the network.

3. Stability Period: the time period from the start of the network operation till the death of the very first node from nodes of an operating WSN.

4. Throughput: the numbers of packets sent to the BS by the nodes in each round. The throughput really shows the effective energy utilization in the network.

5. Packets received: indicate the actual packets acknowledged by BS.

6. Residual Energy: The residual energy of the network helps to analyze the energy consumption of nodes in each round.

Figure 2 shows the number of active sensors during simulation per round in SEP, MGEAR and proposed routing protocol. From the graph, it is clear that HMGEAR has enhanced the lifetime of the network better than the other schemes. In every round of the simulation, the proposed scheme sustains more alive nodes than the two existing protocols until the end of the simulation period. This means that in HMGEAR, nodes stayed alive longer than SEP and MGEAR routing protocols and hence have a better lifetime than the existing protocol. The effective performance of the new protocol is due to the adoption of the multi-hop transmission between cluster heads, gateway, and the BS. This has minimized the rate of data transmission and hence decreases the energy exhaustion in the network.

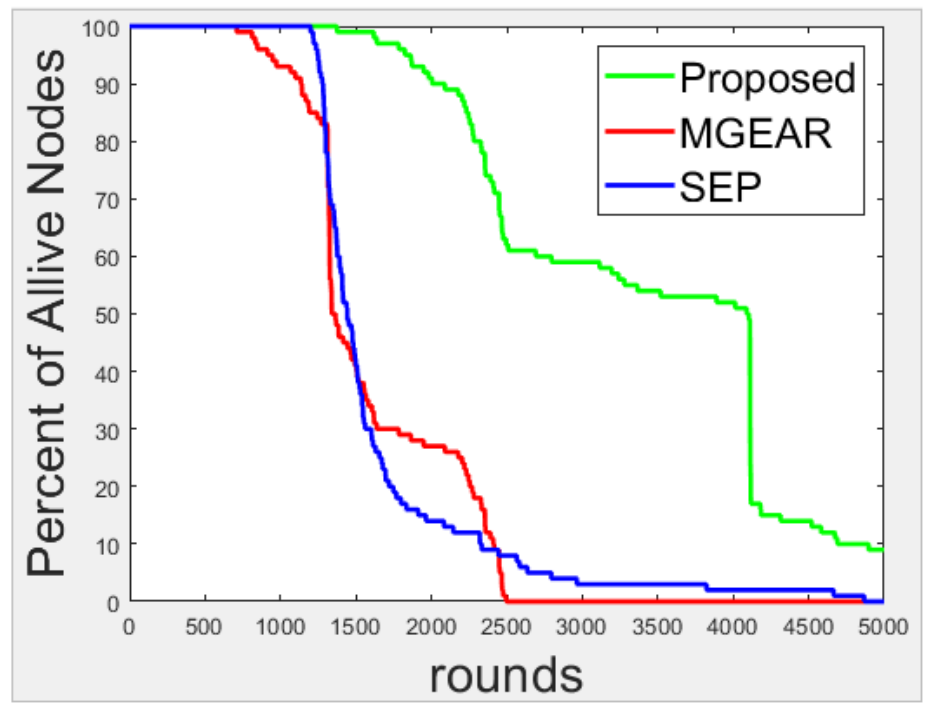

Figure 2. Number of Alive Nodes per round.

Figure 3 also shows the contrast of dead nodes per rounds in the routing protocols. It can be observed that, the new scheme has better stability period than the existing protocol. The existing schemes begin to die earlier compared to the new protocol. However, the new algorithm maintains a low death rate of the nodes until the end of the simulation period followed by SEP protocol. The new scheme and SEP are heterogeneous routing schemes and that is why they have an appreciable stability period. 


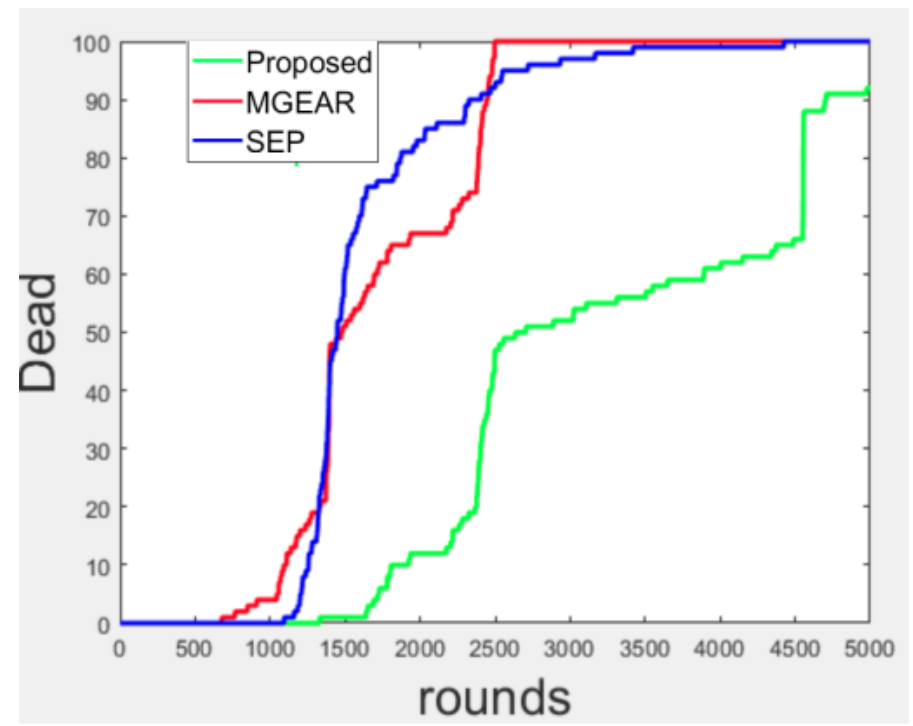

Figure 3. Number of dead Nodes per round.

In Figures 4 and 5, the number of packets forward to the BS and quantity of the packets received by the BS are compared in all the routing algorithms. It was realised that the proposed protocol sent more packets to the Base station and also the BS received more packets from the new scheme compared with the existing schemes as shown in Figures 4 and 5. This is due to the multi-hop communication approach that was introduced in the new protocol. The nodes require less energy to relay their report to the next Region until it gets to the Sink. Also, the gateway node aggregates the report rather than the CHs. This also conserved the energy of the CHs and therefore transmit more data to the gateway node.

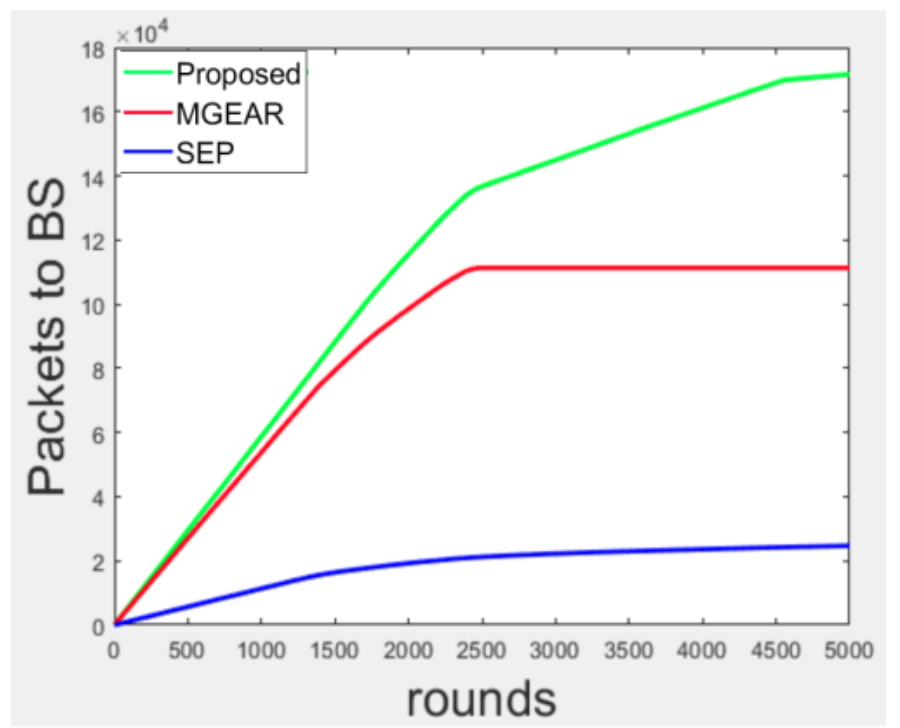

Figure 4. Packets to BS per round. 


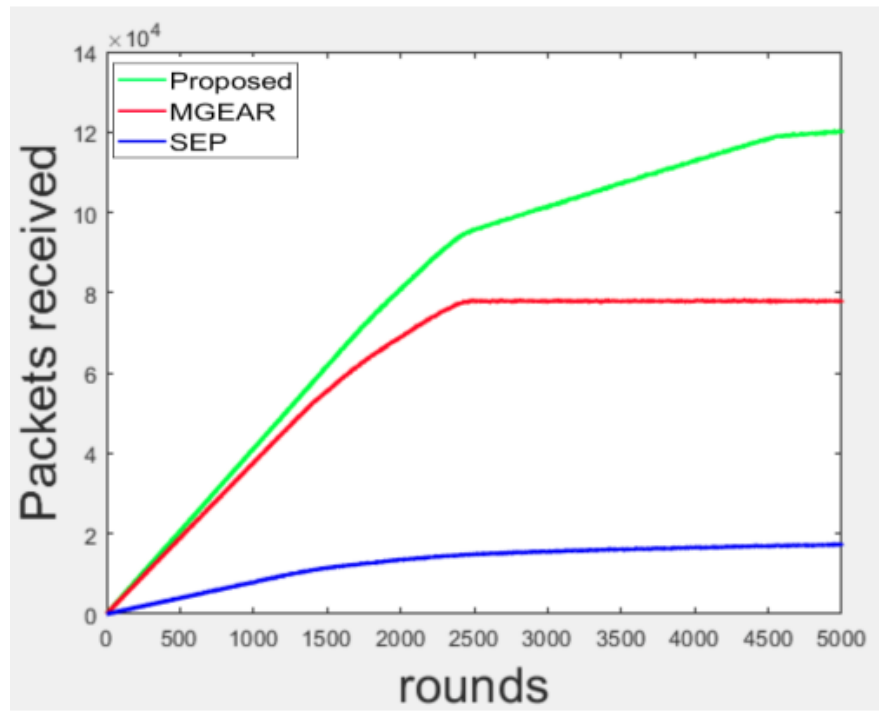

Figure 5. Packets to BS per round.

Figure 6 shows the leftover energy of the sensors per round in all the schemes. It can be noticed that, the rate of energy depletion in the proposed scheme is lower than the existing algorithms. This reflects the energy-conserving mechanisms that were introduced and implemented in the new scheme which includes selecting heads using residual energy of the nodes, multi-hop communication technique employed, and implementation of energy hole removing technique.

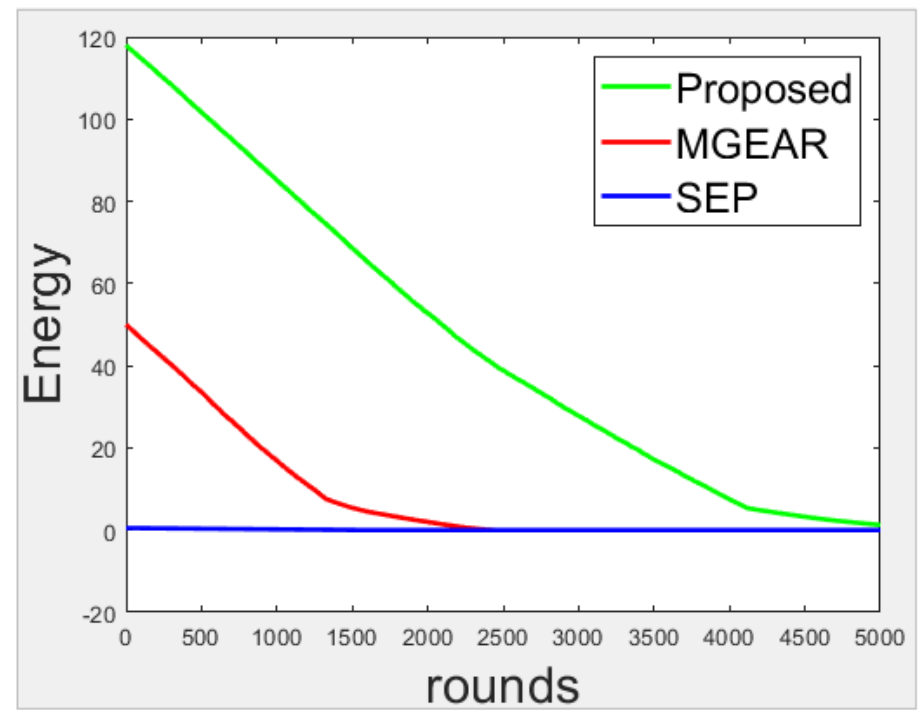

Figure 6. Residual Energy per round.

\section{Conclusion}

A heterogeneous MGEAR routing protocol, HGEAR has been proposed in this paper. The new scheme was assessed with the existing routing algorithms, MGEAR and SEP. The new algorithm introduced heterogeneous nodes in the regions that are far from the Base station as well as multi-hop communication among these nodes in all the regions. The scheme further selects the cluster heads based on the residual energy of the nodes and implements an energy hole removal mechanism. All these were done to reduce the depletion of the sensor nodes in all the regions. A simulation was conducted and results 
showed that the new protocol performed better than the existing schemes in respect to stability period, throughputs, residual energy, and network lifespan.

\section{References}

1. Tuyishimire, E.; Adiel, I.; Rekhis, S.; Bagula, B.A.; Boudriga, N. Internet of things in motion: A cooperative data muling model under revisit constraints. 2016 Intl IEEE Conferences on Ubiquitous Intelligence \& Computing, Advanced and Trusted Computing, Scalable Computing and Communications, Cloud and Big Data Computing, Internet of People, and Smart World Congress (UIC/ATC/ScalCom/CBDCom/IoP/SmartWorld). IEEE, 2016, pp. 1123-1130.

2. Ismail, A.; Bagula, B.A.; Tuyishimire, E. Internet-of-things in motion: A uav coalition model for remote sensing in smart cities. Sensors 2018, 18, 2184.

3. Bagula, A.; Tuyishimire, E.; Wadepoel, J.; Boudriga, N.; Rekhis, S. Internet-of-things in motion: a cooperative data muling model for public safety. 2016 Intl IEEE Conferences on Ubiquitous Intelligence \& Computing, Advanced and Trusted Computing, Scalable Computing and Communications, Cloud and Big Data Computing, Internet of People, and Smart World Congress (UIC/ATC/ScalCom/CBDCom/IoP/SmartWorld). IEEE, 2016, pp. 17-24.

4. Las Fargeas, J.; Kabamba, P.; Girard, A. Cooperative surveillance and pursuit using unmanned aerial vehicles and unattended ground sensors. Sensors 2015, 15, 1365-1388.

5. Tuyishimire, E.; Bagula, A.; Rekhis, S.; Boudriga, N. Cooperative data muling from ground sensors to base stations using UAVs. 2017 IEEE Symposium on Computers and Communications (ISCC). IEEE, 2017, pp. 35-41.

6. Han, G.; Liu, L.; Jiang, J.; Shu, L.; Hancke, G. Analysis of energy-efficient connected target coverage algorithms for industrial wireless sensor networks. IEEE Transactions on Industrial Informatics 2015, 13, 135-143.

7. John, T.O.; Ukwuoma, H.C.; Danjuma, S.; Ibrahim, M. Energy Consumption in Wireless Sensor Network. Energy $2016,7$.

8. Sabri, A.; Al-Shqeerat, K. Hierarchical cluster-based routing protocols for wireless sensor networks-a survey. International Journal of Computer Science Issues (IJCSI) 2014, 11, 93.

9. Tuyishimire, E.; Bagula, A.; Ismail, A. Clustered data muling in the internet of things in motion. Sensors 2019, $19,484$.

10. Tuyishimire, E.; Bagula, B.A.; Ismail, A. Optimal clustering for efficient data muling in the internet-of-things in motion. International Symposium on Ubiquitous Networking. Springer, 2018, pp. 359-371.

11. Heinzelman, W.R.; Chandrakasan, A.; Balakrishnan, H. Energy-efficient communication protocol for wireless microsensor networks. Proceedings of the 33rd annual Hawaii international conference on system sciences. IEEE, 2000, pp. 10-pp.

12. Qing, L.; Zhu, Q.; Wang, M. Design of a distributed energy-efficient clustering algorithm for heterogeneous wireless sensor networks. Computer communications 2006, 29, 2230-2237.

13. Sharma, N.; Verma, V. Heterogeneous LEACH protocol for wireless sensor networks. International Journal of Advanced Networking and Applications 2013, 5, 1825.

14. Elbhiri, B.; Saadane, R.; Aboutajdine, D.; others. Developed Distributed Energy-Efficient Clustering (DDEEC) for heterogeneous wireless sensor networks. 2010 5th International Symposium On I/V Communications and Mobile Network. IEEE, 2010, pp. 1-4.

15. Jibreel, F. Improved-gateway-based energy-aware multi-hop routing protocol for WSNs. International Journal of Innovative Science and Research Technology 2018, 3, 625-630.

16. Saini, P.; Sharma, A.K. Energy efficient scheme for clustering protocol prolonging the lifetime of heterogeneous wireless sensor networks. International Journal of computer applications 2010, 6, 30-36.

17. Jibreel, F. Improved Enhanced Distributed Energy Efficient Clustering (iE-DEEC) scheme for heterogeneous wireless sensor network. International Journal of Engineering Research and Advanced Technology (IJERAT) 2019, 5, 6-11.

18. Jibreel, F.; Jibreel, F. Extended Threshold Stable Election Protocol for Wireless Sensor Networks. North Am. Acad. Res 2019, 2.

19. Jibreel, F.; Gbolagade, K.; Daabo, M. Servant-LEACH Energy Efficient Cluster-Based Routing Protocol for Large Scale Wireless Sensor Network 2018.

20. Jibreel, F. Gateway-based Threshold Distributed Energy Efficient Clustering (G-TDEEC). International Journal of Computer Applications 2019, 182, 43-46.

21. F. Jibreel, M.I. Daabo, K.G. DistanceDistributed Energy Efficient Clustering (D-DEEC) Routing Protocol. The 13th International Multi-Conference on ICT Applications, 2019, pp. 49-53.

22. Kaur, G.; Kaur, S. Enhanced m-gear protocol for lifetime enhancement in wireless clustering system. International Journal of Computer Applications 2016, 147.

23. Jibreel, F.; Daabo, M.; Gbolagade, K. Gateway-stable election protocol for heterogeneous wireless sensor network. Asian Journal of Research in Computer Science 2020, pp. 40-48.

24. Nadeem, Q.; Rasheed, M.B.; Javaid, N.; Khan, Z.A.; Maqsood, Y.; Din, A. M-GEAR: Gateway-based energy-aware multi-hop routing protocol for WSNs. 2013 Eighth international conference on broadband and wireless computing, communication and applications. IEEE, 2013, pp. 164-169.

25. Tuyishimire, E.; Bagula, B.A. A Formal and Efficient Routing Model for Persistent Traffics in the Internet of Things. 2020 Conference on Information Communications Technology and Society (ICTAS). IEEE, 2020, pp. 1-6.

26. Purkar, S.V.; Deshpande, R.S. Energy efficient clustering protocol to enhance performance of heterogeneous wireless sensor network: EECPEP-HWSN. Journal of Computer Networks and Communications 2018, 2018. 